\title{
Leitthema
}

Bundesgesundheitsbl 2014 $\cdot 57: 541-548$

DOI 10.1007/s00103-013-1921-0

Online publiziert: 25. April 2014

(c) Springer-Verlag Berlin Heidelberg 2014

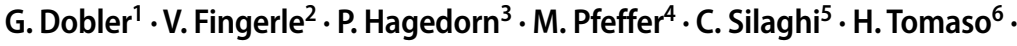 \\ K. Henning ${ }^{7} \cdot$ M. Niedrig ${ }^{3}$ \\ ${ }^{1}$ Institut für Mikrobiologie der Bundeswehr, München \\ ${ }^{2}$ Nationales Referenzzentrum für Borrelien, Dienststelle Oberschleißheim, Bayerisches \\ Landesamt für Gesundheit und Lebensmittelsicherheit (LGL), Oberschleißheim \\ ${ }^{3}$ Zentrum für Biologische Gefahren und Spezielle Pathogene (ZBS-1), Robert Koch-Institut, Berlin \\ ${ }^{4}$ Institut für Tierhygiene und Öffentliches Veterinärwesen, Universität Leipzig \\ ${ }^{5}$ Lehrstuhl für Vergleichende Tropenmedizin und Parasitologie, Veterinärmedizinisches \\ Department, Ludwig-Maximilians-Universität München \\ ${ }^{6}$ Institut für bakterielle Infektionen und Zoonosen, Friedrich-Loeffler- \\ Institut, Bundesforschungsinstitut für Tiergesundheit, Jena \\ ${ }^{7}$ Nationales Referenzlabor für Q-Fieber, Institut für bakterielle Infektionen und Zoonosen, \\ Friedrich-Loeffler-Institut, Bundesforschungsinstitut für Tiergesundheit, Jena
}

\section{Gefahren der Übertragung von Krankheitserregern durch Schildzecken in Deutschland}

Unter den durch blutsaugende Vektoren (Stechmücken, Sandmücken, Fliegen, Flöhe, Läuse, Wanzen, Zecken) übertragenen Infektionserregern haben in Deutschland die durch Zecken übertragenen Erreger die größte medizinische Bedeutung. Auch wenn durch Mücken übertragene Pathogene wie das WestNil-Virus (WNV) in zahlreichen südeuropäischen Ländern (u. a. in Griechenland, Italien) immer wieder für Schlagzeilen sorgen und auch als Bedrohung für Deutschland diskutiert werden, sind die Zecken-übertragenen Infektionserreger offensichtlich weit verbreitet, aber nach wie vor nur unzureichend untersucht.

Infektionskrankheiten wie die LymeBorreliose (LB) und Frühsommer-Meningoenzephalitis (FSME), die durch den Stich der häufigsten Schildzecke in Deutschland, dem Holzbock (Ixodes ricinus), auf den Menschen übertragen werden, sind zwar hinlänglich bekannt, aber es gibt auch hier weiterhin viele offene wissenschaftliche Fragestellungen, die insbesondere das tatsächliche Infektionsrisiko und die geografische Verbreitung der Erreger betreffen. Die derzeitigen Verbreitungskarten spiegeln die wirkliche Präsenz von FSMEV nur un- zureichend wider. Darüber hinaus wurden in neueren Untersuchungen weitere Infektionserreger in Zecken nachgewiesen (• Tab. 1), deren Bedeutung als Gesundheitsgefahr für den Menschen allerdings noch unzureichend geklärt ist. Viren wie das Uukuniemi-Virus (UUKV), Tribec-Virus (TRBV), EyachVirus (EYAV), Erve-Virus (ERVV) oder auch Bakterien wie Francisella tularensis, Anaplasma phagocytophilum, Candidatus Neoehrlichia mikurensis (CNM) oder Coxiella burnetii sind vielen Ärzten nicht bekannt. $\mathrm{Zu}$ den entsprechenden Krankheitsbildern liegen ebenfalls nur wenige bis gar keine Informationen vor. Im vorliegenden Beitrag sollen die aktuellen Erkenntnisse zu diesen neuen Infektionserregern vorgestellt werden, um die Ärzte sowie die Bevölkerung für diese potenziellen Gefahren zu sensibilisieren und weiterführende Untersuchungen anzuregen.

\section{Durch Zecken übertragene Arboviren}

In Deutschland konnte seit Beginn der 1960er-Jahre das autochthone Vorkommen von insgesamt 5 durch Zecken über- tragenen Arboviren direkt oder indirekt nachgewiesen werden [1].

\section{FSME-Virus}

Die wichtigste Arbovirus-Infektion in Mitteleuropa ist die durch Zecken übertragene Frühsommer-Meningoenzephalitis (FSME). Das FSME-Virus (FSMEV) wird in die Familie der Flaviviren eingruppiert. Die Infektion tritt endemisch in Bayern, Baden-Württemberg und Teilen Hessens, Sachsens und Thüringens auf. Aus anderen Bundesländern werden sporadische Erkrankungsfälle gemeldet. Dabei ist unklar, inwieweit es sich hier um wenig aktive Herde oder um neu eingeschleppte und etablierte Naturherde handelt. Etwa jeder 20. FSME-Fall in Deutschland wurde auf Reisen im Ausland erworben (RKI, SurvStat). Jährlich werden zwischen 200 und 500 Erkrankungsfälle registriert (RKI, SurvStat) mit einer hohen Schwankungsbreite, die eine Abhängigkeit der Inzidenz von Wetterbedingungen und anderen ökologischen und soziologischen Faktoren aufzeigt. Die Infektion verläuft mit zunehmendem Alter tendenziell schwerer und führt in bis zu 10\% der Fälle zu bleibenden neurologi- 


\begin{tabular}{|c|c|c|c|}
\hline Infektionserreger & Zeckenspezies & Vorkommen/Verbreitung ${ }^{a}$ & Mögliche Erkrankung \\
\hline $\begin{array}{l}\text { Frühsommer-Meningoenzephalitis- } \\
\text { Virus (FSMEV) }\end{array}$ & Ixodes ricinus & Zentraleuropa, Skandinavien & Enzephalitis \\
\hline Tribec-Virus (TRBV) & Ixodes ricinus & Tschechische Republik & Nicht bekannt \\
\hline Uukuniemi-Virus (UUKV) & Ixodes ricinus & Finnland & Nicht bekannt \\
\hline Eyach-Virus (EYAV) & Ixodes ricinus & Baden-Württemberg & Nicht bekannt \\
\hline Erve-Virus (ERVEV) & Ixodes ricinus & Saarland & Hämorrhagische Enzephalitis \\
\hline $\begin{array}{l}\text { Borrelia burgdorferi sensu lato } \\
\text { ( } 5 \text { humanpathogene Genospezies) }\end{array}$ & $\begin{array}{l}\text { Ixodes ricinus, } \\
\text { Ixodes persulcatus (Osteuropa, Asien), } \\
\text { Ixodes scapularis (USA Nordosten), } \\
\text { Ixodes pacificus (USA Westen) }\end{array}$ & Europa, USA, Asien & $\begin{array}{l}\text { Erythema migrans, Neuroborreliose, } \\
\text { Borrelien-Lymphozytom, Lyme- } \\
\text { Arthritis, Acrodermatitis chronica } \\
\text { atrophicans }\end{array}$ \\
\hline Francisella tularensis & $\begin{array}{l}\text { Ixodes ricinus, Dermacentor margina- } \\
\text { tus, Dermacentor reticulatus }\end{array}$ & Zentraleuropa, Skandinavien & $\begin{array}{l}\text { Schmerzhafte Hautulzeration, } \\
\text { Fieber, regionale Lymphknoten- } \\
\text { schwellung sowie Kopf- und Glieder- } \\
\text { schmerzen, Lungenentzündung, } \\
\text { oropharyngeale Tularämie }\end{array}$ \\
\hline Coxiella (C.) burnetii & Ixodes ricinus, Dermacentor & Deutschland, europaweit & $\begin{array}{l}\text { Q-Fieber, grippeähnliche Erkrankung } \\
\text { mit hohem Fieber, Kopfschmerzen } \\
\text { und Lungenentzündung }\end{array}$ \\
\hline $\begin{array}{l}\text { Rickettsia helvetica, Rickettsia slova- } \\
\mathrm{ca} \text {, Rickettsia raoultii }\end{array}$ & $\begin{array}{l}\text { Ixodes ricinus, Dermacentor reticulatus, } \\
\text { Dermacentor maginatus }\end{array}$ & Europa & $\begin{array}{l}\text { Zeckenbissfieber,",Tick-borne lym- } \\
\text { phadenopathy“ (TIBOLA) }\end{array}$ \\
\hline Anaplasma phagocytophilum & $\begin{array}{l}\text { Ixodes ricinus, Ixodes persulcatus (Ost- } \\
\text { europa, Asien), Ixodes scapularis (USA } \\
\text { Nordosten), Ixodes pacificus (USA } \\
\text { Westen) }\end{array}$ & Europa, USA, Asien & Granulozytäre Anaplasmose \\
\hline $\begin{array}{l}\text { Candidatus Neoehrlichia mikurensis } \\
\text { (CNM) }\end{array}$ & Ixodes ricinus, Ixodes persulcatus & $\begin{array}{l}\text { Deutschland Tschechische Repub- } \\
\text { lik, Schweiz, Dänemark, Schweden }\end{array}$ & $\begin{array}{l}\text { Neoehrlichiose in immunsuppri- } \\
\text { mierten Patienten }\end{array}$ \\
\hline $\begin{array}{l}\text { Babesia microti, Babesia divergens, } \\
\text { Babesia venatorum }\end{array}$ & Ixodes ricinus & Deutschland, europäische Länder & $\begin{array}{l}\text { Hämolyse, Anämie, Ikterus bei im- } \\
\text { munsupprimierten Patienten }\end{array}$ \\
\hline
\end{tabular}

schen Schäden. 1-2\% der gemeldeten Erkrankungsfälle versterben, meist indirekt an Folgeerkrankungen schwerer neurologischer Residuen [2]. Der Naturzyklus des FSMEV, seine Periodizität und seine Pathogenese werden bisher nur sehr unvollständig verstanden.

\section{Tribec-Virus}

Das Tribec-Virus (TRBV) wurde erstmalig in den gleichnamigen Bergen in der ehemaligen Tschechoslowakei nachgewiesen [3]. Es gehört der Gattung Orbivirus innerhalb der Familie Reoviridae an. Aus Tschechien stammen viele Virusisolate, während es in Deutschland in Zecken bisher nur zufällig im Rahmen von FSME-Untersuchungen entdeckt wurde. Auch ist der serologische Nachweis einer Infektion in Hasen in Norddeutschland gelungen [4]. TRBV konnte in der ehemaligen Tschechoslowakei verschiedentlich mit neurologischen Erkrankungen assoziiert werden [5]. Die genaue Ver- breitung des TRBV und seine medizinische Bedeutung in Deutschland sind völlig unbekannt.

\section{Eyach-Virus}

Das Eyach-Virus (EYAV) wurde in einem Waldgebiet nahe dem gleichnamigen Ort in Baden-Württemberg im Rahmen von FSME-Untersuchungen an Zecken isoliert [6]. Es gelang die weitere Isolierung eines sehr ähnlichen Virus in Frankreich [7]. Mittels molekularbiologischer Untersuchungen konnte es am Ort der Originalisolierung in Zecken erneut nachgewiesen werden [8].

Beim EYAV handelt es sich um ein Coltivirus aus der Familie Reoviridae. Der bisher einzige bekannte Verwandte des EYAV ist das Colorado-ZeckenfieberVirus, das nur im Westen der USA vorkommt. In früheren Arbeiten wurde eine Assoziation des EYAV mit einer ZNSSymptomatik gefunden [9]. Die weitere geografische Verbreitung und die medizi- nische Bedeutung des EYAV in Deutschland sind unbekannt.

\section{Uukuniemi-Virus}

Das Uukuniemi-Virus (UUKV) wurde erstmals in Finnland isoliert und identifiziert [10]. Nachdem es lange Jahre als Typvirus für eine eigene Gattung (Uukuvirus) in der Familie Bunyaviridae aufgelistet war, wird es nun in die Gattung Phlebovirus der gleichen Virusfamilie eingruppiert und bildet dort das Typvirus für die Zecken-übertragenen Phleboviren. Auch in Deutschland konnte es zufällig im Rahmen von FSME-Durchseuchungsstudien in Zecken nachgewiesen werden [11]. Es errang in den letzten Monaten wieder eine gewisse Aufmerksamkeit, da 2 neu entdeckte humanpathogene Bunyaviren in China (HuaiyangshanVirus) und in den USA (Heartland-Virus) genetisch mit UUKV nahe verwandt sind. Die Humanpathogenität des UUKV ist ungeklärt. Über seine Verbreitung und 
medizinische Bedeutung in Deutschland liegen bisher keinerlei Daten vor.

\section{Erve-Virus}

Eine weitere Arbovirus-Infektion scheint in Deutschland geografisch sehr begrenzt vorzukommen. Das Erve-Virus (ERVEV) wurde erstmalig aus Spitzmäusen in Frankreich in der Nähe des gleichnamigen Flusses isoliert [12]. Es war dort mit dem Auftreten einer hämorrhagischen Enzephalitis in Zusammenhang gebracht worden. Es handelt sich um ein Virus aus der Thiafora-Gruppe der Gattung Nairovirus in der Familie Bunyavivirdae. Serologisch konnte ein Zirkulieren des Virus außerhalb Frankreichs durch den Nachweis von Antikörpern gegen ERVEV in Nagetieren im Saarland nachgewiesen werden. ERVEV scheint an der Entstehung von starken Kopfschmerzen durch Mikroblutungen im Gehirn beteiligt zu sein [13]. Über die genaue Verbreitung, die Ökologie des Virus und die medizinische Bedeutung dieses mit dem KrimKongo-Hämorrhagischen-Fieber-Virus verwandten Virus in Deutschland und Europa existieren weiter keine Daten. Der direkte Nachweis von ERVEV in Zecken ist bislang nicht gelungen, aber das Vorkommen in diesen aufgrund seiner taxonomischen Zugehörigkeit zum Genus Nairovirus ist anzunehmen, da bislang alle bekannten Vertreter dieses Genus von Zecken übertragen werden.

\section{Durch Zecken übertragene Bakterien}

\section{Borrelia burgdorferi s.l. - Erreger der Lyme-Borreliose}

Mit der Entdeckung des Erregers Borrelia burgdorferi und der Zusammenführung bis dahin ätiologisch unklarer Krankheitsbilder zur nosologischen Entität „Lyme-Borreliose“ (LB) eröffnete sich die Möglichkeit einer spezifischen Diagnostik und kausalen Therapie von Erkrankungen aus praktisch allen medizinischen Fachdisziplinen. Die LB gilt mittlerweile als die häufigste durch vektorübertragene Erreger ausgelöste Erkrankung in den gemäßigten Klimazonen der nördlichen Hemisphäre. Ihr Ver-

Bundesgesundheitsbl 2014·57:541-548 DOI 10.1007/s00103-013-1921-0

(c) Springer-Verlag Berlin Heidelberg 2014

\section{G. Dobler $\cdot$ V. Fingerle $\cdot$ P. Hagedorn · M. Pfeffer $\cdot$ C. Silaghi $\cdot$ H. Tomaso $\cdot$ K. Henning $\cdot$ M. Niedrig \\ Gefahren der Übertragung von Krankheitserregern durch Schildzecken in Deutschland}

\section{Zusammenfassung}

Durch Zecken übertragbare Krankheitserreger haben eine große Bedeutung für die Gesundheit der deutschen Bevölkerung. Viren wie das Frühsommer-MeningoenzephalitisVirus (FSMEV), das Uukuniemi-Virus, das Tribec-Virus und das Eyach-Virus oder Bakterien wie Borrelien, Rickettsien, Francisella tularensis, Anaplasma phagocytophilum, Candidatus Neoehrlichia mikurensis (CNM) oder Coxiella burnetii wurden in der in Deutschland häufigsten Zecke, der Schildzecke Ixodes ricinus, nachgewiesen. Während allgemein bekannt ist, dass Zecken FSMEV und Borrelien übertragen können, ist selbst in Fachkreisen nicht bekannt, dass sie auch die anderen oben genannten Erreger tragen und ggf. entsprechende Infektionen verursachen können. Auch wenn in Deutschland in Einzelfällen Erkrankungen nach Übertragung der zuletzt genannten Erreger durch Zecken bereits beschrieben wurden, fehlen systematische Untersuchungen über ihr Vorkommen und ihre pathogenen Eigenschaften. So ist weitgehend unklar, welche Bedeutung neu entdeckte Infektionserreger wie Candidatus Neoehrlichia mikurensis oder auch lange bekannte Erreger wie Rickettsien spielen, die sich bisweilen in einem Fünftel der untersuchten Zecken nachweisen lassen. Ob Klimaänderungen zur weiteren Ausbreitung der Zecken und der Infektionserreger beitragen, bedarf ebenfalls weiterer Untersuchungen. Die Initiativen zur Schaffung natürlicher Lebensräume und der Trend zum häufigeren Aufenthalt in der Natur im Rahmen von Freizeitaktivitäten verstärkt die Gefahr, in Kontakt mit Zecken und ihren Infektionserregern zu kommen. Folglich kann in Zukunft mit dem Auftreten weiterer, bisher nicht bekannter Erkrankungen durch die Übertragung diese Erreger gerechnet werden.

Schlüsselwörter

Zecken-übertragene Arboviren · LymeBorreliose - Rickettsiose - Anaplasmose . Rückfallfieber

\section{Threat of transmission of infectious pathogens by Ixodes ricinus ticks in Germany}

\section{Abstract}

Tick-transmitted diseases are of great importance for the general health of the German population. Several viruses, such as tickborne encephalitis virus (TBEV), Uukuniemi virus, Tribec virus, Eyach virus or bacteria, such as Borrelia, Rickettsiae, Francisella tularensis, Anaplasma phagocytophilum, Candidatus Neoehrlichia mikurensis (CNM) and Coxiella burnetii were detected in the most prominent tick in Germany, the hard tick /xodes ricinus. While infections, such as TBE and Lyme disease are well known, other infections are hardly known even among experts. Although there have been a few descriptions of isolated cases in Germany, a systematic investigation regarding the distribution and the pathogenic potential of these pathogens is still lacking. In particular elderly people and people with underlying diseases seem to be mostly affected. The impor- tance of new infectious disease agents, such as Candidatus Neoehrlichia mikurensis but also of long known pathogens, such as Rickettsiae still remains unclear, while some of them could be detected in $20 \%$ of investigated ticks. Whether climate change contributes to the further distribution of these infectious agents remains unclear and requires further investigation. The increasing initiatives to create natural environments and the trend towards spending more time in nature for recreational activities will increase the danger of coming into contact with ticks and the respective infectious agents. Considering these circumstances an increase of diseases caused by these pathogens is to be expected.

\section{Keywords}

Tick transmitted diseases - Lyme disease . Rickettsiosis · Anaplasmosis · Relapsing fever breitungsgebiet deckt sich mit dem Vorkommen ihrer Vektoren, Schildzecken aus dem I.-ricinus-/I.-persulcatus-Komplex. In Deutschland sind etwa $1 \%$ der
Larven, 10\% der Nymphen und 20\% der adulten Zecken Borrelienträger. Von den mittlerweile 18 beschriebenen B.-burgdorferi-sensu-lato-Genospezies sind 5 
gesichert humanpathogen und auch in Deutschland vorhanden: B. burgdorferi sensu stricto, B. afzelii, B. garinii, B. bavariensis und B. spielmanii [14].

Die klinischen Manifestationen dieser Multisystemerkrankung können außerordentlich vielfältig sein und betreffen insbesondere die Haut, Gelenke, das Nervensystem und das Herz [15]. Am häufigsten ist das nach Tagen bis Wochen um den Zeckenstich sich zentrifugal ausbreitende Erythema migrans (Wanderröte). Die häufigste disseminierte frühe Form ist die Meningoradikulitis Bannwarth mit heftigsten, nachtsbetonten Schmerzsyndromen und Hirnnervenparesen. Späte Erkrankungen - mit Inkubationszeiten von Monaten bis Jahren - können als Lyme-Arthritis die großen Gelenke mit mono- bis oligoartikulären voluminösen Ergüssen betreffen, die Haut als Acrodermatitis chronica atrophicans mit papierdünner Epidermis und Abbau von Binde- und Fettgewebe („Zigarettenpapierhaut") und das Nervensystem mit vielfältigsten neurologischen Symptomen (Enzephalomeningomyelitis), die u. a. auch einer multiplen Sklerose ähneln können.

Bei einer prospektiven, populationsbasierten Studie im Raum Würzburg fand sich eine Inzidenz der Lyme-Borelliose von 111/100.000 Einwohner und Jahr, was auf Deutschland hochgerechnet etwa 90.000 neuen Fällen pro Jahr entspricht [16]. Bei 89\% der Patienten fand sich ein Erythema migrans, bei 3\% eine Neuroborreliose, bei $2 \%$ ein Lymphozytom, bei $<1 \%$ eine Karditis, bei $5 \%$ eine Lyme-Arthritis, bei $1 \%$ eine Acrodermatitis chronica atrophicans und eine chronische Neuroborreliose in keinem Fall. Eine Studie, basierend auf Krankenkassendaten, geht von wenigsten 215.000 LymeBorelliose-Fällen/Jahr in Deutschland aus [17], während Daten der länderspezifischen Meldepflicht (östliche Bundesländer) maximal 30.000 Fälle in Deutschland erwarten lassen [18].

Insgesamt gilt die LB mittels einer Antibiotikagabe über 2 bis 3 Wochen als gut therapierbar [15], jedoch sind weitere gut kontrollierte Studien zum Therapieerfolg und zur Langzeitprognose speziell disseminierter Manifestationen anzustreben.
Prophylaktische Maßnahmen beschränken sich derzeit auf das Vermeiden von Zeckenstichen, - d. h. das Meiden zeckendurchseuchter Gebiete, die Anwendung von Repellenzien und das Tragen geeigneter Kleidung - bzw. die frühzeitige Zeckenentfernung, da die Wahrscheinlichkeit der Übertragung von Borrelien mit der Dauer des Saugaktes steigt. Eine Impfung, die die genetisch heterogene Borrelienpopulation in Deutschland berücksichtigt, ist derzeit in der Entwicklung.

Insgesamt muss die derzeitige Datenlage zur Epidemiologie sowie auch zu einzelnen Aspekten der Diagnostik und Therapie der LB als unzureichend bezeichnet werden. Für die Aufklärung des Bürgers, die Risikokommunikation, die Implementierung von Präventionsmaßnahmen und für gezieltere Therapieempfehlungen sind belastbarere Daten - z. B. über Sentinel- oder populationsbasierte Studien - dringend zu erheben.

\section{Borrelia miyamotoi - Erreger des Rückfallfiebers}

Erst kürzlich wurde B. miyamotoi als Erreger humanen Rückfallfiebers nachgewiesen. Die Patienten zeigten ein unspezifisches, Influenza-artiges Erkrankungsbild u. a. mit Fieber (z. T. auch Fieberrückfälle), Muskel-, Gelenk- und Kopfschmerzen. Einige Patienten hatten auch ein Erythema migrans [19]. Die nach molekulargenetischen Analysen den Rückfallfieber-Borrelien zugeordnete B. miyamoto $i$ wird über dieselben Schildzecken wie die LB übertragen, bei uns durch I. ricinus. Relevante Studien aus Deutschland zur Häufigkeit und geografischen Ausbreitung dieser Borrelie oder zur Inzidenz der Erkrankung fehlen weitestgehend. In Deutschland wurden bisher keine Erkrankungsfälle nachgewiesen. Nachdem die ersten 2 Fälle aus Italien und Holland bekannt wurden, sollte diese Erkrankung bei der Diagnose berücksichtigt werden, insbesondere bei Influenza-artigen Symptomen nach Zeckenstich.

\section{Francisella tularensis-} Erreger der Tularämie

Tularämie (Hasenpest, Hirschfliegenfieber, Ohara-Krankheit) ist eine durch das Bakterium Francisella tularensis verursachte Zoonose. Derzeit werden 4 Subspezies unterschieden: F. tularensis tularensis, F. tularensis holarctica, F. tularensis mediaasiatica und $F$. tularensis novici$d a$. In Deutschland wurde bisher nur die Subspezies F. tularensis holarctica isoliert, die einen etwas leichteren Krankheitsverlauf verursacht, als die in Nordamerika vorkommende Subspezies F. tularensis tularensis [20].

Die Übertragung des Erregers kann durch Haut- oder Schleimhautkontakt mit infektiösem Tiermaterial, Verzehr von nicht ausreichend erhitztem, kontaminiertem Fleisch (Hasen), Wasser, Inhalation von infektiösem Staub oder durch den Stich blutsaugender Parasiten (Zecken, Mücken, Stechfliegen) erfolgen. Die Infektionsdosis ist mit ca. 10 bis 50 Bakterien sehr niedrig, weshalb auch immer wieder Laborinfektionen vorkommen. Die Inkubationszeit beträgt in der Regel 3 bis 5 Tage.

Der Krankheitsverlauf und die Symptome beim Menschen sind von der durch die Subspezies bestimmten Virulenz des Erregers sowie von seiner Eintrittspforte in den Körper abhängig. Die häufigste klinische Form ist die ulzeroglanduläre Tularämie mit schmerzhafter Hautulzeration an der Eintrittspforte, Fieber, regionaler Lymphknotenschwellung sowie mit Kopf- und Gliederschmerzen. Wichtig sind weiter die oropharyngeale Form (Beläge und Geschwüre im Pharynx und an den Tonsillen, Lymphknotenschwellung), die okuloglanduläre Form (meist einseitige Konjunktivitis, Lidödem, Lymphknotenschwellung) und die schwer verlaufende pulmonale Form.

In den letzten Jahren wurden in Deutschland nur einstellige Fallzahlen gemeldet; auf der Halbinsel Eiderstedt in Nordfriesland gab es aber in den Jahren 1950/1951 und 1957/1958 Ausbrüche mit jeweils über 100 Erkrankten. Die meisten Tularämiefälle beim Menschen werden in Deutschland durch den Kontakt mit oder den Verzehr von infizierten Feldhasen verursacht. Bei Feldhasen verläuft die Tu- 
larämie zumeist tödlich, wobei die Tiere zunächst lethargisch werden und Krankheitsherde in inneren Organen (z. B. Leber, Lunge, Milz) auftreten.

Das natürliche Reservoir der Bakterien und die Rolle von Vektoren bei ihrer Übertragung sind noch nicht ausreichend untersucht. Kleine Nagetiere sterben rasch an der Infektion und dienen vermutlich kaum als Reservoir [21]. Es gibt Hinweise darauf, dass Feldhasen selten auch chronisch infiziert sein können und dann als Infektionsquellen fungieren. Francisellen können auch in Amöben überleben und über kontaminiertes Wasser Menschen und Tiere infizieren. In Skandinavien spielen infizierte Stechmücken und Stechfliegen eine relevante Rolle, in Deutschland hingegen kaum. Hier wurden Francisellen in Ixodes ricinus, Dermacentor marginatus und D. reticulatus nachgewiesen [22]. In Studien von Tomaso und Kollegen wurden die Bakterien aus Zecken isoliert, die von infizierten Hasen gesammelt wurden. Eine Rolle der Zecken als Vektor für Francisellen ist damit aber nicht bewiesen. Selbst in Zecken (I. ricinus) von Vögeln, die kaum empfindlich gegenüber Francisellen sind, konnte deren DNA mittels PCR nachgewiesen werden [23]. So ist es vorstellbar, dass Francisellen mit Zugvögeln über weite Strecken transportiert werden.

Phylogenetische Studien und Ausbruchsuntersuchungen von $F$. tularensis holarctica sind dadurch erschwert, dass sich zwischen einzelnen Isolaten kaum genetische Unterschiede finden lassen. Bei Isolaten aus Feldhasen in Deutschland konnten bisher nur 3 Genotypen unterschieden werden, wobei ein großer Cluster im Nordosten vorherrscht, der andere im Südwesten [24]. Die zukünftige Sequenzierung von Gesamtgenomen in größerem Umfang wird voraussichtlich eine genauere Differenzierung ermöglichen.

\section{Coxiella burnetii - Erreger des Q-Fiebers}

Das Q-Fieber ist in Deutschland eine meldepflichtige humane Erkrankung und eine meldepflichtige Tierkrankheit. Die Q-Fieber-Infektion wird durch das obligat intrazellulär wachsende Bakte- rium Coxiella (C.) burnetii hervorgerufen. In den letzten Jahren wurde in den Medien vermehrt über Q-Fieber-Ausbrüche in Deutschland und in den Niederlanden berichtet. Beim Q-Fieber handelt es sich um eine Zoonose, d. h. um eine Infektionskrankheit, die vom Tier auf den Menschen übertragen wird. Oftmals gehen die Erkrankungsfälle beim Menschen von erkrankten Schaf- und Ziegenbeständen aus, aber auch Rinder können die Quelle der Infektion darstellen. Beim Menschen löst der Erreger eine grippeähnliche Erkrankung mit hohem Fieber, Kopfschmerzen und Lungenentzündung aus. Die Übertragung erfolgt überwiegend durch das Inhalieren erregerhaltiger Aerosole und kontaminierter Stäube und kann über weite Strecken stattfinden. Des Weiteren wird der Erreger auch durch Zecken übertragen. So konnte C. burnetii in mehr als 40 Zecken-Spezies nachgewiesen werden [25]. Hierzu gehört auch der internationale Referenzstamm „Nine Mile“, der ebenfalls aus einer Zecke isoliert wurde [26]. Die höchste Erregerkonzentration findet sich im ZeckenKot [25], wodurch besonders bei der Schafschur eine Gefährdung von Personen ausgeht. Bei den einheimischen Zecken scheinen nur die beiden Dermacentorarten eine Rolle zu spielen [27]. Dagegen ist die Bedeutung des Holzbocks für die Verbreitung des Q-Fiebers vermutlich vernachlässigbar gering, wie Untersuchungen in den Niederlanden ergaben. Dort konnte Coxiella-DNA nur dann in von Schafen gesammelten Zecken nachgewiesen werden, wenn diese kurz zuvor mit einer Q-Fieber-Vakzine geimpft worden waren [28].

Wie die Literatur zeigt, waren in den meisten Fällen Wiederkäuer die Quelle für humane Q-Fieber-Erkrankungen. Zecken stellen allerdings ein Reservoir für den Erreger dar, wodurch der Wildtierzyklus und somit die Quelle für mögliche Neuinfektionen der Nutztiere aufrechterhalten wird. Hierzu sind noch eingehende Untersuchungen erforderlich.

Rickettsia spp.- Erreger der Rickettsiosen

Die ausschließlich intrazellulär lebenden Bakterien der Gattung Rickettsia gehören zur Klasse der $\alpha$-Proteobacteria. Dort werden sie in der Familie der Rickettsiaceae innerhalb der Ordnung der Rickettsiales angesiedelt Es sind bislang 6 Arten dieser Gattung in Zecken aus Deutschland nachgewiesen worden [29]. Die häufigste hiervon ist Rickettsia helvetica, die ein Zeckenbissfieber hervorrufen kann. In Schweden, Frankreich, der Schweiz und in Italien wurden klinische Fälle bekannt, die durch eine Infektion mit $R$. helvetica hervorgerufen wurden. Die Symptome variieren stark und umfassen eine Perimyokarditis und Fieber mit und ohne Ausschlag [30]. Welche Rolle dieses Pathogen bei anderen Erkrankungen spielt, ist unklar. Diskutiert werden in diesem Zusammenhang unter anderem Fazialisparesen und plötzliche Taubheit [31]. Ähnliches gilt für die ebenfalls häufigen Rickettsienarten $R$. slovaca und $R$. raoultii, die mit der Tick-borne lymphadenopathy (TIBOLA) assoziiert werden. Diese bislang ausschließlich in Dermacentorarten vorkommenden Rickettsienarten könnten mit der räumlichen Ausbreitung von $D$. reticulatus [32] und möglicherweise auch D. marginatus an Bedeutung gewinnen. Die ebenfalls in Deutschland nachgewiesene Rickettsienart $R$. monacensis wurde als möglicher Auslöser einer dem Mittelmeerfleckfieber ähnlichen Erkrankung beschrieben [33].

\section{Anaplasma phagocytophilum - Erreger der Anaplasmose}

Wie die Rickettsien gehört auch Anaplasma phagocytophilum zu der in der Klasse der a-Proteobacteria angesiedelten Ordnung der Rickettsiales. In dieser Ordnung befindet sich die Familie Anaplasmataceae mit der Gattung Anaplasma und $\operatorname{der}$ Art A. phagocytophilum. Es handelt sich ebenfalls um ein intrazelluläres Bakterium, das beim Menschen vor allem neutrophile Granulozyten infiziert und sich in ihnen vermehrt. In Mitteleuropa wird es durch I. ricinus übertragen. Es löst beim Menschen die humane granulozytäre Anaplasmose (HGA) aus. Die Leitsymptome umfassen Fieber, Krankheitsgefühl, Muskelschmerzen, Kopfschmerzen, Übelkeit, Erbrechen und Husten. Mehr als ein Drittel der Patienten weist ein makulopapulöses Exan- 
them auf. Häufig zeigt sich ein Rigor. Obwohl die Prävalenz dieses Pathogens in Zecken in Deutschland zwischen 0 und 8,7\% [34] schwankt, sind hier bislang keine klinischen Fälle beim Menschen bekannt. Dieses ist umso erstaunlicher, als in Österreich schon 2006 klinische Fälle beschrieben wurden [35]. Erkrankungen ausgelöst durch $A$. phagocytophilum treten auch bei Hunden, Pferden, Rindern und Schafen auf und wurden bereits in Deutschland beschrieben. Der Grund für die Diskrepanz zwischen dem Vorkommen von A. phagocytophilum in Zecken und Wildtieren (bis zu annähernd $100 \%$ in einer untersuchten Rehpopulation) und dem Fehlen klinischer Fälle beim Menschen ist nicht bekannt [36]. Damit ist die tatsächliche Bedeutung der Anaplasmose beim Menschen in Deutschland bisher weder ausreichend geklärt noch umfassend untersucht.

\section{Candidatus Neoehrlichia \\ mikurensis - Erreger \\ der Neoehrlichiose}

Ende der 1990er-Jahre wurden von einer Forschergruppe DNA-Sequenzen in Ixodes-Zecken gefunden, die mit einer generischen DNA-Sonde für Ehrlichien hybridisierten, aber mit keiner der verwendeten Spezies-spezifischen DNA-Sonden für die damals bekannten Ehrlichienarten reagierten. Man fand diese taxonomisch nicht gruppierbare EhrlichienDNA in 19 von 121 vollgesaugten I. ricinus, die von 1998 in Holland erlegten Rehen stammten [37]. Bei einer Untersuchung von 357 I. ricinus, die zwischen 1998 und 2001 von asymptomatischen italienischen Patienten gesammelt wurden, wurde diese DNA in 10 Zecken gefunden. Für den entsprechenden Erreger wurde dann der Name Candidatus Ehrlichia walkeri sp. nov. vorgeschlagen [38]. Auch in Deutschland wurden im Jahr 2003 erstmals entsprechende DNA-Sequenzen in I. ricinus beschrieben [39]. Nachdem der Erreger 2003 auch in China nachgewiesen wurde, erschien $1 \mathrm{Jahr}$ später die Arbeit einer japanischen Gruppe, die die DNA des „,neuen“ Erregers in 7 von 15 Wanderratten (Rattus norvegicus) und in 5 von 128 einzeln oder in Pools getesteten I. ovatus auf Hokkaido nachwies [40]. Die Isolierung des Bakteriums gelang bisher nicht, phylogenetische Analysen führten zu der derzeit gültigen taxonomischen Stellung und Bezeichnung Candidatus Neoehrlichia mikurensis (CNM). Außer in den bereits erwähnten Ländern wurde CNM in unterschiedlichen Prävalenzen in Zecken und/ oder Nagern auch in zahlreichen europäischen Staaten (Schweiz, Tschechische Republik, Frankreich, Schweden, Dänemark) nachgewiesen. Die derzeit bekannte geografische Verbreitung reicht somit von Japan im äußersten Osten Asiens über den nördlichen Teil des asiatischen Kontinentes bis nach Italien und Frankreich in Europa [41].

Die Tatsache, dass es auch seither nicht gelungen ist, CNM in vitro in gängigen Zellkultursystemen zu propagieren oder mit anderen Methoden außerhalb von Wirtstieren zu vermehren, erklärt, warum hier nur wenig bekannt ist. Man weiß nur, dass es sich um ein obligat intrazelluläres, Gram-negatives Bakterium mit endothelialem Zelltropismus handelt. Bezüglich des Vektors konnte CNM bislang ausschließlich in Zecken der Gattung Ixodes gefunden werden [42].

Im Jahr 2007 wurde CNM bei einem 69-jährigen Mann mit immunsuppressiver Therapie nach Verdacht auf eine chronisch entzündliche, demyelinisierende Polyneuropathie sowie $1 \mathrm{Jahr}$ später (2008) bei einem 57-Jährigen mit intrazerebralen und subarachnoidalen Blutungen und einem Aneurysma an der Carotis interna diagnostiziert [41]. In den Jahren 2008 und 2009 wurden 2 Fälle bei ebenfalls immunkomprimierten Patienten in der Tschechischen Republik gefunden [41].

2009 wurden in der Schweiz und in Schweden 2 weitere Patienten mit Neoehrlichiose diagnostiziert, die ebenfalls eine Grunderkrankung hatten [41]. Bis zum Oktober 2012 waren diese 6 Fälle die einzigen labordiagnostisch bestätigten humanen Neoehrlichiosen. Dies änderte sich durch einen Bericht aus China: Dort wurde bei 7 von 622 Fieberpatienten im Nordosten des Landes CNM mittels PCR im Blut nachgewiesen [43]. Die derzeit letzten Fallberichte stammen aus der Schweiz, wo 2 Patienten nach entsprechender Doxycycline-Behandlung schnell fieberfrei wurden und eine Restitutio ad integrum gelang [44].

\section{Babesia spp. - Erreger \\ der Babesiose}

Babesien werden zur Ordnung Piroplasmida gezählt, in der die Familie Babesiidae enthalten ist. Die Gattung Babesia enthält über 100 Babesienarten, die meist sehr wirtsspezifisch sind. Sporozoiten der Babesien befallen die Erythrozyten von Wirbeltieren. Eine Übertragung der Sporozoiten ist bereits nach einem Tag mit dem Zeckenspeichel möglich. Der Befall des Ovars und damit der Eier der Zecke stört die Entwicklung der Zeckenlarven nicht. Das bedeutet, dass Zecken das Reservoir für Babesien bilden, in dem sie auch ohne Vorhandensein von Blutwirten mehrere Generationen existieren können.

An der Entstehung einer symptomatischen Babesiose ist maßgeblich die Immunantwort des Wirtes beteiligt. In mild verlaufenden Babesiosen wird die Bildung von Cytokinen [z. B. von Tumornekrosefaktor- $\alpha$ (TNF- $\alpha$ ) oder von Interleukin 6] hochreguliert. Es wird vermutet, dass eine überschießende Produktion dieser Stoffe zu einer Pneumonie führt. Der Befall der Erythrozyten löst darüber hinaus eine Hämolyse aus, was direkt zu einer Anämie und einem Ikterus führen kann.

In Deutschland spielt die Babesienspezies Babesia divergens (rinderassoziiert) eine Rolle bei immunsupprimierten Patienten. Die Krankheitsfälle sind meist schwerer, als bei den bisher seltenen Fällen, die durch B. microti (nagerassoziiert) ausgelöst wurden. Babesia venatorum (bisher nur in Rehwild nachgewiesen) kann nur molekularbiologisch von B. divergens unterschieden werden. Daher ist unklar, ob und wie viele der vor der Entwicklung molekularbiologischer Methoden beschriebenen humanen Babesiosefälle auf diese Babesienart zurückzuführen sind.

Der erste Fall von humaner Babesiose wurde in Kroatien beschrieben [45]. Er trat bei einer immunsupprimierten Person auf. Der erste Fall einer humanen Babesiose bei einer immunkompetenten Person fand sich auf der Nantucket 
Island vor der Küste von Massachusetts [46]. Seitdem wurden Fälle im Nordosten und im höheren mittleren Westen der USA berichtet. In Japan und Taiwan verursachten Babesia-microti-ähnliche Erreger Erkrankungen, und in Südkorea wurde ein neuer Babesienstamm (KO1) identifiziert. Sporadische Fälle der Babesiose traten aber auch in Afrika (Ägypten), Australien, und Südamerika (Kolumbien) auf [47, 48]. Im Jahr 2007 wurde der erste autochthone Fall von Babesiose in Westeuropa (Deutschland) beschrieben [49].

\section{Fazit}

Auch wenn die Zahl der Berichte über einzelne Fälle von Anaplasmose, Babesiose, Infektionen mit Candidatus Neoehrlichia mikurensis und Tularämie in Deutschland und angrenzenden Ländern leicht angestiegen sind, fehlen noch wesentliche Erkenntnisse über das Vorkommen dieser Infektionserreger in diesen Regionen und über ihre mögliche gesundheitliche Bedeutung. Trotz der über die letzten 10 Jahre deutlich verbesserten diagnostischen Methoden stehen systematische Untersuchungen zur Prävalenz und geografischen Verbreitung dieser Infektionserreger in Zecken in Deutschland noch aus. Die einzelnen Fallberichte zeigen, dass Personen mit Vorerkrankungen oder Immunsupprimierte ein deutlich höheres Risiko haben, entsprechende Krankheitssymptome zu entwickeln. Dies ist ein Aspekt, der aufgrund des immer größer werdenden Anteils älterer Menschen an der Gesamtbevölkerung an Bedeutung gewinnen wird. Ohne weitere umfangreichere Untersuchungen zu Verbreitung, Dynamik und zu den Pathogenitätsfaktoren von durch Zecken übertragenen Infektionserregern werden wir unsere Kenntnisse auch zukünftig nur aus den wenigen diagnostisch bestätigten Einzelfällen beziehen können. Inwieweit von den berichteten neuen Erregern ein weit größerer Personenkreis betroffen ist, der nach Zeckenstich mit unklarer Symptomatik gar nicht oder ggf. auch falsch behandelt wird, lässt sich aufgrund der mangelnden Erkenntnisse bisher nicht einmal abschätzen. Aus Sicht des „Pub- lic Health" wäre es daher durchaus sinnvoll, die Bedeutung dieser derzeit in Zecken nur seltenen nachgewiesenen Infektionserreger, d. h. ihre Verbreitung und ihr pathogenes Potenzial für den Menschen, eingehender zu untersuchen, um eine realistische Abschätzung der gesundheitlichen Gefahren zu ermitteln [50].

\section{Korrespondenzadresse}

\section{Prof. Dr. M. Niedrig}

Zentrum für Biologische Gefahren und Spezielle Pathogene (ZBS-1),

Robert Koch-Institut

Nordufer 20, 13353 Berlin

niedrigm@rki.de

Danksagung. Für die Anregungen und Unterstützung bei diesem Beitrag möchten wir uns recht herzlich bei Prof. Jabbar Ahmed, Borstel, und Prof. Heiner Neubauer, Jena, bedanken.

\section{Einhaltung ethischer Richtlinien}

Interessenkonflikt. G. Dobler, V. Fingerle, P. Hagedorn, M. Pfeffer, C. Silaghi, H. Tomaso, K. Henning und M. Niedrig geben an, dass kein Interessenkonflikt besteht.

Dieser Beitrag beinhaltet keine Studien an Menschen oder Tieren.

\section{Literatur}

1. Scheid W, Ackermann R, Bloedhorn H et al (1964) Untersuchungen über das Vorkommen der Zentraleuropäischen Enzephalitis in Süddeutschland. Dtsch Med Wochenschr 89:2313-2317

2. Kaiser R (1999) The clinical and epidemiological profile of tick-borne encephalitis in southern Germany 1994-98: a prospective study of 656 patients. Brain 122:2067-2078

3. Libikova H, Rehaccek J, Gresikova M et al (1964). Cytopathic viruses isolated from Ixodes ricinus ticks in Czechoslovakia, Acta Virol 8:96

4. Dobler G, Wölfel R, Schmuser H et al (2006) Seroprevalence of tick-borne and mosquito-borne arboviruses in European brown hares in Northern and Western Germany. Int J Med Microbiol 296(Suppl 40):80-83

5. Malkova D, Holubova J, Kolman JM et al (1980) Antibodies against some arboviruses in persons with various neuropathies. Acta Virol 24:298

6. Rhese-Küpper B, Casals J, Rhese E, Ackermann R (1976) Eyach - an arthropod-borne virus related to Colorado tick fever virus in Federal Republic of Germany. Acta Virol 20:339-344

7. Chastel C, Main AJ, Couatar-Manac'HA et al (1984) Isolation of Eyach virus (Reoviridae, Colorado tick fever group) from Ixodes ricinus and Ixodes ventalloi ticks in France. Arch Virol 82:161-171
8. Hassler D, Oehme R, Kimmig P, Dobler G (2003) Eyach Virus: Erstmaliger Nachweis aus Zecken nach mehr als 25 Jahren in Südwest-Deutschland. Dtsch Med Wochenschr 128(37):1874

9. Chastel C (1998) Erve and Eyach: two viruses isolated in France, neuropathogenic for man and widely distributes in Western Europe. Bull Acad Natl Med 182:801-810

10. Oker-Blom N, Salminen A, Brummer-Korvenkontio $\mathrm{M}$ et al (1964) Isolation of some viruses other than typical tick-borne encephalitis virus from Ixodes ricinus ticks in Finland. Ann Med Exp Biol Fenn 42:109-112

11. Süss J, Schrader C (2004) Durch Zecken übertragene humanpathogene und bisher als apathogen geltende Mikroorganismen in Europa. Bundesgesundheitsbl Gesundheitsforsch Gesundheitsschutz 47:392-404

12. Wössner R, Grauer MT, Langenbach J et al (2000) The Erve virus: possible mode of transmission and reservoir. Infection 28:164-166

13. Treib J, Dobler G, Haass A et al (1997) Thunderclap headache caused by Erve virus. Neurology 50:509-511

14. Fingerle V, Schulte-Spechtel UC, Ruzic-Sabljic E et al (2008) Epidemiological aspects and molecular characterization of Borrelia burgdorferi s.l. from southern Germany with special respect to the new species Borrelia spielmanii sp. nov. Int J Med Microbiol 298:279-290

15. Stanek G, Fingerle V, Hunfeld KP et al (2011) Lyme borreliosis: clinical case definitions for diagnosis and management in Europe. Clin Microbiol Infect 17:69-79

16. Huppertz HI, Bohme M, Standaert SM et al (1999) Incidence of Lyme borreliosis in the Wurzburg region of Germany. Eur J Clin Microbiol Infect Dis 18:697-703

17. Muller I, Freitag MH, Poggensee G et al (2012) Evaluating frequency, diagnostic quality, and cost of Lyme borreliosis testing in Germany: a retrospective model analysis. Clin Dev Immunol 59:5427

18. RKI (2010) Lyme-Borreliose: Analyse der gemeldeten Erkrankungsfälle der Jahre 2007 bis 2009 aus den sechs östlichen Bundesländern. Epidemiol Bull 12:101-107

19. Platonov AE, Karan LS, Kolyasnikova NM et al (2011) Humans infected with the relapsing fever spirochete Borrelia miyamotoi, Russia. Emerg Infect Dis 17:1816-1823

20. Ellis J, Oyston PC, Green M, Titball RW (2002) Tularemia. Clin Microbiol Rev 15:631-646

21. Kaysser P, Seibold E, Mätz-Rensing K et al (2008) Re-emergence of tularemia in Germany: presence of Francisella tularensis in different rodent species in endemic areas. BMC Infect Dis 17:157

22. Gehringer $\mathrm{H}$, Schacht $\mathrm{E}$, Maylaender $\mathrm{N}$ et al (2013) Presence of an emerging subclone of Francisella tularensis holarctica in Ixodes ricinus ticks from south-western Germany. Ticks Tick Borne Dis 4:93-100

23. Franke J, Fritzsch J, Tomaso H et al (2010) Coexistence of pathogens in host-seeking and feeding ticks within a single natural habitat in Central Germany. Appl Environ Microbiol 76:6829-6836

24. Müller W, Hotzel H, Otto P et al (2013) German Francisella tularensis isolates from European brown hares (Lepus europaeus) reveal genetic and phenotypic diversity. BMC Microbiol 13:61 
25. Široký P, Kubelová M, Modrý D et al (2010) Tortoise tick Hyalomma aegyptium as long term carrier of $\mathrm{Q}$ fever agent Coxiella burnetii-evidence from experimental infection. Parasitol Res 107:1515-1520

26. Samuel JE, Frazier ME, Mallavia LP (1985) Correlation of plasmid type and disease caused by Coxiella burnetii. Infect Immun 49(3):775-779

27. Sting R, Breitling N, Oehme R, Kimmig P (2004) Studies on the prevalence of Coxiella burnetii in sheep and ticks of the genus Dermacentor in $\mathrm{Ba}$ den-Württemberg. Dtsch TierarztI Wochenschr 111(10):390-394

28. Sprong H, Tijsse-Klasen E, Langelaar M et al (2012) Prevalence of Coxiella burnetii in ticks after a large outbreak of $\mathrm{Q}$ fever. Zoonoses Public Health 59(1):69-75

29. Dobler G, Pfeffer M (2012) Spotted fever rickettsiae and rickettsioses in Germany. In: Mehlhorn $\mathrm{H}$ (Hrsg) Arthropods as vectors of emerging diseases. Parasitol Res Monographs 3:361-376

30. Oteo JA, Portillo A (2012) Tick-borne rickettsioses in Europe. Ticks Tick Borne Dis 3:271-278

31. Nilsson $K$, Wallménius $K$, Hartwig $S$ et al (2013) Bell's palsy and sudden deafness associated with Rickettsia spp. infection in Sweden. A retrospective and prospective serological survey including PCR findings. Eur J Neurol. doi:10.1111/ene.12218 [Epub ahead of print]

32. Dautel H, Dippel C, Oehme R et al (2006) Evidence for an increased geographical distribution of Dermacentor reticulatus in Germany and detection of Rickettsia sp. RpA4. Int J Med Microbiol 296(40):149-156

33. Madeddu G, Mancini F, Caddeo A et al (2012) Rickettsia monacensis as cause of Mediterranean spotted fever-like illness, Italy. Emerg Infect Dis 18(4):702-704

34. Silaghi C, Woll D, Hamel D et al (2012) Babesia spp. and Anaplasma phagocytophilum in questing ticks, ticks parasitizing rodents and the parasitized rodents - analyzing the host-pathogen-vector interface in a metropolitan area. Parasit Vectors 5:191

35. Walder G, Fuchs D, Sarcletti M et al (2006) Human granulocytic anaplasmosis in Austria: epidemiological, clinical, and laboratory findings in five consecutive patients from Tyrol, Austria. Int J Med Microbiol 296(40):297-301

36. Overzier E, Pfister K, Herb I et al (2013) Detection of tick-borne pathogens in roe deer (Capreolus capreolus), in questing ticks (Ixodes ricinus), and in ticks infesting roe deer in southern Germany. Ticks Tick Borne Dis 4:320-328

37. Schouls LM, Van De Pol I, Rijpkema SG, Schot CS (1999) Detection and identification of Ehrlichia, Borrelia burgdorferi sensu lato, and Bartonella species in Dutch Ixodes ricinus ticks. J Clin Microbiol 37:2215-2222

38. Sanogo YO, Parola P, Shpynov S et al (2003) Genetic diversity of bacterial agents detected in ticks removed from asymptomatic patients in northeastern Italy. Ann NY Acad Sci 990:182-190

39. Loewenich FD von, Baumgarten BU, Schröppel K et al (2003) High diversity of ankA sequences of Anaplasma phagocytophilum among Ixodes ricinus ticks in Germany. J Clin Microbiol 41:50335040

40. Kawahara M, Rikihisa Y, Isogai E et al (2004) UItrastructure and phylogenetic analysis of "Candidatus Neoehrlichia mikurensis" in the family Anaplasmataceae, isolated from wild rats and found in Ixodes ovatus ticks. Int J Syst Evol Microbiol 54:1837-1843
41. Jahfari S, Fonville M, Hengeveld P et al (2012) Prevalence of Neoehrlichia mikurensis in ticks and rodents from North-west Europe. Parasit Vectors $5 \cdot 74$

42. Richter D, Matuschka FR (2012) "Candidatus Neoehrlichia mikurensis", Anaplasma phagocytophilum, and lyme disease spirochetes in questing european vector ticks and in feeding ticks removed from people. J Clin Microbiol 50:943-947

43. Li H, Jiang JF, Liu W et al (2012) Human infection with Candidatus Neoehrlichia mikurensis, China. Emerg Infect Dis 18:1636-1638

44. Maurer FP, Keller PM, Beuret C et al (2013) Close geographic association of human neoehrlichiosis and tick populations carrying Candidatus Neoehrlichia mikurensis in Eastern Switzerland. J Clin Microbiol 51:169-176

45. Skrabalo Z, Deanovic Z (1957) Piroplasmosis in man; report of a case. Doc Med Geogr Trop 9:1116

46. Spielman A (1976) Human babesiosis on Nantucket Island: transmission by nymphal Ixodes ticks. Am JTrop Med Hyg 25:784-787

47. El-Bahnasawy MM, Khalil HH, Morsy TA (2011) Babesiosis in an Egyptian boy aquired from pet dog, and a general review. J Egypt Soc Parasitol 41:99-108

48. Senanayake SN, Paparini A, Latimer M et al (2012) First report of human babesiosis in Australia. Med J Aust 196(5):350-352

49. Hildebrandt A, Hunfeld KP, Baier M et al (2007) First confirmed autochthonous case of human Babesia microti infection in Europe. Eur J Clin Microbiol Infect Dis 26:595-601

50. Schmidt K, Dressel KM, Niedrig M et al (2013) Public health and vector-borne diseases - a new concept for risk governance. Zoonoses Public Health 60(8):528-538
Wie können wir die Patientensicherheit verbessern?

Das Thema Patientensicherheit hat in der Gesundheitsversorgung in den letzten Jahren zunehmend an Bedeutung gewonnen.

Der offene Umgang und Austausch über unerwünschte Ereignisse wird jedoch bislang eher selten gepflegt, obwohl Studien zeigen, dass in Kliniken in denen ein Fehlerwarnsystem ( reporting systems", CIRS) genutzt wird, die Rate an unerwünschten Ereignissen abnimmt.

Das Leitthemenheft "Patientensicherheit" von Der Unfallchirurg (Ausgabe 10/2013) regt in folgenden Beiträgen dazu an, sich mit dem Thema auseinander zu setzen, bekannte Möglichkeiten zu nutzen, aber auch über weitere Möglichkeiten nachzudenken:

- Patientensicherheit in der Versorgungsforschung

- Das Aufklärungsgespräch als Teil der Patientensicherheit in der Kindertraumatologie

- Was bedeutet Sicherheit im Krankenhaus?

- "Human factors" und "crisis resource management"

- Verbessern simulatorbasierte Teamtrainings die Patientensicherheit?

Bestellen Sie diese Ausgabe zum Preis von 36,- EUR zzgl. Versandkosten bei Springer Customer Service Center Kundenservice Zeitschriften Haberstr. 7, 69126 Heidelberg

Tel.: +49 6221-345-4303

Fax: +49 6221-345-4229

E-Mail: leserservice@springer.com

Suchen Sie noch mehr zum Thema? Mit e.Med, dem Online-Paket von Springer Medizin, können Sie schnell und komfortabel in über 500 medizinischen Fachzeitschriften recherchieren. Weitere Infos unter springermedizin.de/ eMed. 